\title{
CRÍTICA A LA NOCIÓN DE MODELO DE PATRICK SUPPES ${ }^{1}$
}

\author{
Rodrigo López-Orellana \\ Universidad de Salamanca \\ rodrigo.lopez@usal.es \\ Juan Redmond \\ Universidad de Valparaíso \\ juan.redmond@uv.cl
}

\begin{abstract}
RESUMEN / ABSTRACT
Nuestro objetivo es analizar críticamente el enfoque de la noción de modelo de Alfred Tarski que Patrick Suppes integra en su concepción semanticista de las teorías científicas. En efecto, mostraremos cómo esta noción se configura en Suppes $(1969 ; 1970)$ desde una revisión formal de la noción de modelo en Tarski (1936), focalizando nuestro análisis en tres ejes principales: i) una crítica a la perspectiva estática del semanticismo; ii) un análisis de las condiciones restrictivas y el lenguaje para la axiomatización de teorías; y iii) la revisión de las nociones de 'sistema axiomático', 'sistema formal' y 'método axiomático'.
\end{abstract}

Palabras Clave: modelo, sistema axiomático, teoría, semántica estática, Suppes, Tarski.

\section{CRITICISM OF PATRICK SUPPES'NOTION OF MODEL}

Our aim is to critically analyse the approach to the notion of model by Alfred Tarski that Patrick Suppes integrates into his semanticist conception of scientific theories. Indeed, we will show how this notion is configured in Suppes (1960; 1970) from a formal review of the notion of model in Tarski (1936), focusing our analysis on three main axes: i) a critique of the static perspective of semanticism; ii) an analysis of the restrictive conditions and the language for the axiomatization of theories; and iii) the revision of the notions of 'axiomatic system', 'formal system' and 'axiomatic method'.

KEYWORDS: model, axiomatic system, theory, static semantics, Suppes, Tarski.

Este trabajo ha sido financiado por la Agencia Nacional de Investigación y Desarrollo / Proyecto Postdoctorado 2021 No 3210531. Agradezco a María Manzano, Universidad de Salamanca (España), por sus comentarios y sugerencias. 


\section{Introducción: la noción de modelo del semanticismo}

RA Nuestro punto de partida, como señala Moulines $(2011,107)$, es que a partir de los años 50 del siglo XX se abre una nueva fase de la filosofía de la ciencia caracterizada por la centralidad de la teoría formal de modelos de Alfred Tarski (1936) y su aplicación a las ciencias empíricas por parte de Patrick Suppes y sus colaboradores. En efecto, el objetivo que nos proponemos en el presente artículo es el de analizar críticamente el enfoque de la noción de modelo de Alfred Tarski que Suppes integra en su concepción semanticista de las teorías. Mostraremos entonces cómo esta noción se configura en Suppes $(1969 ; 1970)$, desde una revisión formal de la noción de modelo en Tarski (1936), focalizando nuestro análisis en tres ejes principales: i) la semántica estática; ii) las condiciones restrictivas y el lenguaje para la axiomatización de teorías; y iii) la revisión de las nociones de 'sistema axiomático', 'sistema formal' y 'método axiomático'. A tal efecto, no entraremos a revisar detalles históricos ni conceptuales de las distintas propuestas que conforman lo que suele llamarse 'la familia semanticista' (Lorenzano 2008, p. 27), pero suponemos que la noción de modelo de Tarski de 1936 es la noción de base que opera y sustenta a la propuesta de Suppes y que se extiende a todo el enfoque semanticista de las teorías científicas.

Llamaremos aquí como 'básica' a esta noción de modelo porque tanto el estructuralismo metateórico, desarrollado por Joseph D. Sneed (1971) -entre otros-y sistematizado por Wolfgang Balzer, C. Ulises Moulines y J. D. Sneed (1987), como sus modificaciones $^{2}$-v. g. el realismo estructural de John Worrall (1989) o el de Newton C.A. da Costa y Steven French (2003)- comparten esta misma noción de Suppes; además de compartir una misma idea de 'representación'.

La concepción semanticista en filosofía de la ciencia fue iniciada por Suppes en la década de los sesenta $(1960 ; 1962)$, pero tomó forma a partir de la década siguiente con los trabajos del propio Suppes $(1970 ; 1974 ; 1989 ; 1993)$. A él le siguieron diversos filósofos que adoptaron su concepción: Stegmüller (1970; 1973); Bas C. van Fraassen (1980); Maria Luisa Dalla Chiara S. y Giuliano Toraldo di Francia (1973); Balzer, Moulines y Sneed (1987); Frederick Suppe (1989); da Costa y French (2003); entre otros.

2 Cabe advertir que las variantes son diversas, pero pueden identificarse en un conjunto gracias a la idea de representación que ellas comparten. Sin embargo, existen algunos casos como Ronald Giere $(1988 ; 1999)$ que, aunque a veces se le incluye dentro de la familia semanticista, es posible excluirlo de esta caracterización debido a que en su propuesta los modelos de una teoría se conciben de una manera bastante más amplia e informal, atendiendo a los usos que los científicos hacen del término y a la relación de representación con la cual se ligan diferentes tipos de modelo de una misma teoría. De todas maneras, el perspectivismo de Giere comparte con el estructuralismo que las teorías empíricas tienen un componente proposicional fundamental, que en su caso llama 'hipótesis empírica' (Giere 1988, 85), que determina la efectividad de la representación de un modelo sobre los fenómenos del mundo que trata. 
Todos ellos configuran sus propuestas a partir de diferentes posiciones epistemológicas pero que redundan en una misma idea central, esta es:

[...] presentar una teoría no es presentar una clase de axiomas, las teorías no se identifican metateóricamente con conjuntos de enunciados; presentar una teoría es presentar una clase de modelos, las teorías se identifican metateóricamente como conjuntos de modelos (Díez y Moulines 2016, p. 344).

Esto quiere decir que una teoría ya no debe identificarse como un conjunto cerrado de proposiciones (los axiomas) respecto de la relación de consecuencia lógica, como se afirmaba en la concepción clásica de las teorías -'Concepción Heredada', o 'concepción sintáctica de las teorías'-, condensada en las propuestas de Rudolf Carnap (1956) y Carl Gustav Hempel (1962; 1970). Para la perspectiva clásica, una teoría debía identificarse con una clase única y específica de axiomas, de tal manera que cualquier otra clase diferente de axiomas equivale a una teoría completamente distinta a la primera. Contra esta tesis se elaborará la crítica de la concepción semántica según la cual sí podemos concebir intuitivamente que dos axiomatizaciones diferentes, con el mismo apartado conceptual, sean de una misma teoría. La cuestión estriba en que tales axiomatizaciones diferentes, que hablan de una porción determinada del mundo, especifican las mismas relaciones empíricas que se establecen en esa porción de la realidad, esto es, ambas determinan la misma clase de modelos o realizaciones:

Lo que importa de una teoría, lo que la identifica, es lo que dice sobre el comportamiento de determinada parcela de la realidad, no cómo lo dice. Lo esencial es que caracteriza ciertos trozos de la realidad como comportándose de cierto modo. Esto es, que determina ciertos modelos [...] Lo importante es pues qué modelos determina una teoría, no los recursos lingüísticos que emplea para ello (Díez y Moulines 2016, pp. 345-346).

La concepción clásica, a la luz de la perspectiva semanticista, no ofrece una explicación satisfactoria de cómo dos estructuras lingüísticas diferentes pueden componer una misma teoría. Esta entiende que una teoría empírica es el conjunto de las consecuencias de la unión de dos conjuntos diferentes de proposiciones: el de los postulados teóricos y el de las reglas de correspondencia. Pero justamente, según como lo muestran José A. Díez y C. Ulises Moulines, apelar a las consecuencias (lógicas) es apelar implícitamente a modelos: "un enunciado es consecuencia de otros si todos los modelos de estos son modelos de aquél" (Díez y Moulines 2016, p. 346). La mejor vía para comprender cómo dos axiomatizaciones es de una misma teoría es apelando a sus realizaciones, a sus modelos. Es justamente en este punto que se vuelve necesario revisar la noción de 'modelo' que Tarski presentó a partir de su teoría de la verdad, cuya formalización matemática es considerada el pilar fundamental donde descansa la teoría de los modelos lógico-matemática hasta hoy (Hodges 2014). Esta es la noción básica de modelo que subyace a la concepción semanticista. 


\subsection{La noción de modelo de Tarski}

En primer lugar, debe advertirse que la noción de modelo de Tarski que aquí analizamos -y en la que se apoya Suppes- es la que publicó en 1936 en las Actas del Congreso Internacional de Filosofía Científica, celebrado en París en 1935 (Tarski 1936). La versión más conocida de esta idea aparece en su artículo posterior publicado en inglés 'The semantic conception of truth and the foundations of semantics', de 1944. La noción a la que nos referimos es la que se conoce con el nombre de 'modelo de un sistema axiomático'; no es la definida posteriormente a partir de la década de los años 50. Esta aclaración no es comúnmente advertida en filosofía y creemos que es necesario hacerla, no solo por cuestiones del estudio de la semántica y la filosofía de la lógica, sino porque que también ayuda a fijar la propuesta filosófica de Suppes.

Según Tarski, la definición de verdad de una oración, específicamente del lenguaje formal, está determinada por su estructura formal o esquema. La caracterización de dicha estructura cobra sentido a partir de la necesidad de un criterio de adecuación material para la definición de verdad, en el que Tarski insiste constantemente en su obra $(1935 ; 1936 ; 1944 ; 1983)$. Formula una solución a los problemas de la definición lógica de verdad a partir de su 'esquema T' (Scheme-T) (Tarski 1944, §4). Dicho esquema consiste en la siguiente construcción: primero, consideremos una oración arbitraria (cualquiera); luego la reemplazamos por la letra ' $p$ '. Formaremos el nombre de esta oración y lo reemplazaremos por otra letra, por ejemplo ' $X$ '. Nos preguntaremos entonces cuál es la relación lógica que existe entre las dos oraciones ' $X$ es verdadera' y ' $p$ '. Desde el punto de vista de una concepción básica de la verdad, se establecerá una relación de equivalencia. Esto es:

Xes verdadero si, y solo si, $p$.

Tarski llama 'equivalencia de la forma (T)' a toda equivalencia de esta clase, en la que ' $p$ ' sea reemplazada por cualquier oración del lenguaje a que se refiere la palabra 'verdadero', y ' $X$ ' sea reemplazada por un nombre de esta oración (Tarski 1944, p. 344). De esta manera, según Tarski, se puede formular de una forma más precisa las condiciones en que consideraremos el uso y la definición del término 'verdadero' como adecuado desde el punto de vista material:

[...] deseamos usar el término 'verdadero', de manera tal que puedan enunciarse todas las equivalencias de la forma (T), y llamaremos 'adecuada' a una definición de la verdad si de ella se siguen todas estas equivalencias (Tarski 1944, p. 344).

Es importante señalar que Tarski propone esta definición de verdad solo para lenguajes formales, no para el uso natural o corriente de verdad.

La definición de Tarski es una definición eliminativa de la verdad, que trata de incorporar el concepto en un metalenguaje que no contenga conceptos semánticos ambiguos y que nos indica, de forma sistemática, cómo reemplazar las apariciones de la expresión 'verdadera' por otras expresiones que se suponen más claras. Además, la 
equivalencia del esquema de oración $(\mathrm{T})$ solo es una definición parcial de la verdad, ya que explica solo en qué consiste la verdad de esa oración específica, de su tipo específico de estructura. Una definición general o definitiva de la verdad debe ser una conjunción lógica de todas las definiciones parciales (Tarski 1944, pp. 344-346).

Tarski propone el nombre de 'concepción semántica de la verdad' para su teoría. Para él, la semántica "se ocupa de ciertas relaciones entre las expresiones de un lenguaje y los objetos o 'estados de cosas' a los que se 'refieren' esas expresiones”' (Tarski 1944, 345). Las nociones semánticas -tales como designación, satisfacción y definiciónson las que expresan ese tipo de relaciones. De este modo, 'verdadero' expresará una propiedad (o denotará una clase) de ciertas expresiones: las oraciones o sentencias declarativas. Tendrá entonces una naturaleza lógica diferente: 'verdadero' no es más que un predicado de esas expresiones. En efecto, -como señalamos anteriormente- a Tarski solo le interesaba definir los 'predicados de verdad' para esa clase de entidades que son las sentencias declarativas y, específicamente, para lenguajes formales (de primer orden). Las tesis centrales que ofreció acerca de la definición de verdad son:

i. una oración es verdadera si refiere o designa un estado de cosas existente;

ii. usaremos 'verdadero' de tal manera que puedan enunciarse todas las equivalencias de las expresiones que tienen el mismo esquema o forma de una oración (declarativa); esto gracias al criterio de adecuación material de la definición; y

iii. la definición misma de verdad, y todas las equivalencias implicadas por ella, han de formularse en el metalenguaje (Tarski 1944, p. 350).

Con esta última tesis (iii.) Tarski $(1944, \S 9)$ propuso su conocida distinción entre metalenguaje y lenguaje objeto. No desarrollaremos esta distinción, ya que no viene al caso para este trabajo. Pero, en relación a la idea de 'semántica' que tienen los llamados 'semanticistas', solo conviene recordar que con esta distinción se trata el problema de la verdad especificando los requisitos o condiciones lógicas para la estructura de estos dos lenguajes. Ahora bien, i. y ii. no se refieren a las oraciones mismas sino a los objetos acerca de los que hablan las oraciones, o a los estados de cosas descritas por ellas. En este sentido, lo importante es entonces lograr establecer adecuadamente la relación que existe entre esos dos tipos diferentes de entidades, las oraciones y los objetos/estados-de-cosas-del-mundo. En definitiva, la pregunta central será cuál es la relación entre el lenguaje y el mundo del cual habla.

Para resolver el problema, Tarski recurre a otro tipo de entidades: los modelos (Tarski 1944), pp. 348-369). En efecto, la estructura lógica que ha especificado -el esquema de oración $(\mathrm{T})$ - se entenderá como un modelo posible del lenguaje. Y un lenguaje (formal) tiene muchas interpretaciones -realizaciones- posibles, que son sus modelos posibles. La conexión entre lenguaje y modelo está garantizada por la definición misma de verdad, que determina para cada oración y estructura si la oración es falsa o verdadera en la estructura: es el puente que conecta el lenguaje formal con sus interpretaciones posibles por medio de modelos. Como sabemos, un lenguaje 
formal es un lenguaje construido artificialmente para expresar lo que se quiere decir sobre ciertas estructuras lógicas o matemáticas que representan la realidad. Por ejemplo -y como lo hace Tarski-, aplicando su definición de verdad y la teoría de conjuntos podemos reemplazar la realidad por ciertas estructuras conjuntistas que en cierto modo la representan (v. g., si tomamos el conjunto de los números naturales y sus relaciones). Podríamos ascender del lenguaje natural que describe esa realidad a un lenguaje formalizado dentro de la teoría de conjuntos -resolviendo las vaguedades del compromiso de 'lo verdadero' de sus expresiones a cuestiones de naturaleza puramente deductivas dentro del mismo marco de la teoría-. Lo dicho en ese lenguaje formal, sus expresiones, son ahora estructuras matemáticas que toman el papel de la realidad y la modelan, la representan.

Bajo esta idea, la teoría de modelos sería el estudio de las relaciones entre lenguajes y sus interpretaciones o realizaciones posibles. En un sentido amplio, se ha entendido también como el estudio de las interpretaciones de lenguajes cualesquiera, naturales o formales, mediante estructuras conjuntistas y siguiendo el paradigma del esquema (T). Entonces, el esquema abstracto de la teoría de modelos es el siguiente.

Tenemos un lenguaje formal $L$ y una clase de objetos $K$, que son los modelos, y entre ambos se tiende un puente: la noción de verdad.

A partir de todo esto, para Tarski 1) un modelo matemático será entonces una representación aritmética de una teoría empírica, esto es, un conjunto de proposiciones matemáticas que poseen la misma forma que las leyes de la teoría. Por ello se dice que se establece un isomorfismo estructural entre la representación -el modelo- y la teoría. Y, 2) un modelo lógico debe entenderse como una interpretación semántica de un sistema de axiomas (del cálculo lógico de una teoría) tales que los axiomas son verdaderos para dichas interpretaciones, que no son necesariamente entidades lingüísticas, pero deben ser isomorfos en su estructura lógica a la teoría (Tarski 1977, caps. V-VI).

En la terminología matemática después de Tarski, y también en la terminología de las concepciones modelo-teoréticas en filosofía de la ciencia, se prefiere hablar de 'realización' del lenguaje $L$ o de estructura asociada a $L$ antes que de 'interpretación ' de un lenguaje $L$. En lógica impera el concepto de interpretación. Pero la idea básica de interpretación de un sistema es como sigue. El criterio de adecuación de la definición de verdad exige que, por ejemplo, si tenemos el siguiente enunciado de un lenguaje $L$ :

$$
\text { El coatí es un plantígrado, }
$$

su interpretación en $L$ se cumple cuando dicho individuo o especie (el coatí), que a su vez es la referencia de dicho nombre, tiene la propiedad que expresa el enunciado (ser 'plantígrado' o 'animal que apoya completamente la planta del pie para andar'), que es a su vez el significado del predicado. Decimos entonces que esta relación se cumplirá cuando: para toda interpretación en $L$, que representamos mediante un par ordenado $<U, m>$, donde $U$ es el conjunto no vacío que representa el universo de discurso de $L$ y $m$ es la operación que asigna significados en $U$ a las constantes descriptivas de $L$, por extensión, $m$ asocia un significado a una constante individual. De este modo, esta relación 
establece una vinculación con un individuo (o individuos) de un universo - por este motivo algunos hablan de una 'realización del lenguaje' en un sistema determinado-. Y, según Tarski, el significado que $m$ asocia al predicado del argumento será el conjunto de individuos de $U$.

\subsection{La interpretación de Suppes}

Ahora, Suppes (1960) supuso que esta concepción de la noción de modelo de Tarski era la base para la significación del concepto tanto para las disciplinas formales como para las factuales -se trataba del mismo significado-. Afirmó que la definición estándar lógico-matemática de modelo puede usarse como 'concepto fundamental' para todas las demás disciplinas, la diferencia está en el uso que hacen del concepto. Propuso, entonces, una constancia de significado pero una diferencia de uso determinada por los distintos objetos de estudio en las diferentes disciplinas científicas, formales o empíricas (Suppes 1960, p. 289). El concepto de modelo de Tarski representa para Suppes una definición estándar para las ciencias naturales gracias a sus nociones técnicas de realización posible y de representación, que han sido señaladas anteriormente.

Entonces, según la definición de Tarski, un modelo es 'una realización posible en que una teoría es satisfecha'. Y una realización posible de una teoría es 'una entidad conjuntista del tipo lógico adecuado'. Así, una teoría es una entidad lingüística consistente de un conjunto de enunciados. Un modelo será una entidad no-lingüística, una estructura o sistema, constituido por entidades de diverso tipo, en la que la teoría es satisfecha (Suppes 1960, p. 289). Y según la teoría de la verdad especificada en el esquema (T), la teoría será verdadera en dicho sistema si 'el modelo realiza la teoría'. El modelo de la teoría es una realización posible de esta ya que en el sistema que caracteriza es posible que "pase lo que la teoría dice" (Díez y Moulines 2016, p. 297).

Ahora bien, la estrategia es aplicar la teoría de conjuntos (aplicación que realiza el mismo Tarski): el modelo reemplaza a esa realidad de la cual trata, que aparecerá entonces caracterizada por ciertas estructuras conjuntistas que en cierto modo la representan. De esta manera, un modelo se entiende como un cierto tipo de tupla ordenada consistente de un conjunto de objetos y relaciones y operaciones sobre esos objetos de la realidad en cuestión (Suppes 1960, pp. 290-291).

Siguiendo a Tarski, Suppes introducirá la noción de modelo como 'posible realización conjuntista'. Con ello pretende redefinir el lugar que ocupa la axiomatización como método más adecuado para una caracterización más rica y pertinente de las teorías científicas, especialmente para aquellas que revisten modelos más complejos o teorías que tienen un contenido de conocimiento más rico o más interesante, más allá del recurso formal clásico de la lógica de primer orden, como lo es el caso de la mecánica de partículas -como lo muestran Díez y Moulines 2016, pp. 350-351)-. El enfoque que inaugura Suppes entiende que " [...] axiomatizar una teoría es definir un predicado en términos de nociones de teoría de conjuntos [predicado conjuntista]" (Suppes 1957, p. 249). Sus seguidores afirmarán que esta idea permite comprender mejor cómo funcionan los modelos y cómo logran ser capturados por el nuevo tipo de axiomatización propuesta (Frigg 2006; Lorenzano 2013). 
La introducción del predicado teórico-conjuntista, definido en términos de la teoría de conjuntos -específicamente, del cálculo intuitivo de conjuntos-, servirá para responder la pregunta clásica acerca de cuáles deben ser los elementos específicos de una teoría. Con este predicado se define la clase de modelos de una teoría y es del tipo (Díez y Moulines 2016, pp. 350-351):

' $x$ es un sistema $\operatorname{syss}_{d e f} \varphi(x)^{\prime}$

Donde $\varphi$ especifica:

i. las entidades de $x$ y el conjunto de relaciones entre ellas y sus funciones - de la estructura-;

ii. las condiciones definicionales: que especifican los tipos lógicos de las entidades de $x$ (si se trata de dominios de objetos, de relaciones o funciones), su constitución relativa (dominios y contradominios de relaciones y funciones), y sus propiedades matemáticas generales, cuyas caracterizaciones se realizan a través de axiomas impropios (sui generis). Este tipo de axiomas determinan el tipo de entidades, las propiedades matemáticas y las relaciones lógicas de constitución de la estructura en cuestión; y

iii. las condiciones restrictivas no lógicas de la estructura: los axiomas en sentido propio. Aquí aparecen -específicamente- las leyes de la teoría, cuyo efecto constrictivo inmediato es que solo algunas estructuras que satisfacen las condiciones definicionales de ii, podrán ahora satisfacer las condiciones especificadas por los axiomas propios.

Suppes (1954, p. 245) describe entonces su método axiomático en cuatro pasos:

1. Debe hacerse explícito qué teorías se están asumiendo junto con la teoría en cuestión. Por ejemplo, la teoría de gases de Amedeo Avogadro -específicamente, la ley de Avogadro ${ }^{3}$ - supone la aceptación inicial de la teoría atómica de John Dalton y la teoría de Louis Joseph Gay-Lussac -específicamente, la ley sobre los vectores de movimiento en la molécula, o 'ley de Gay-Lussac'-.

2. Es necesario señalar o enumerar las nociones primitivas de la teoría y especificar su carácter conjuntista. Por ejemplo, para el caso de la mecánica de partículas: el conjunto de partículas, el intervalo de tiempo, la función de posición y la función de masa ${ }^{4}$.

3 "Volúmenes iguales de gases distintos bajo las mismas condiciones de presión y temperatura, contienen el mismo número de moléculas" (Morselli 1984, pp. 89-90).

$C f r$. el ejemplo de Suppes para: ' $x$ es un sistema de mecánica (newtoniana) de partículas syss $_{d e f}$ existen $P, T, s, m, f$ (Díez y Moulines 2016, p. 351). 
3. Debe completarse la definición teórico-conjuntista enumerando los axiomas que deben satisfacerse, especialmente los axiomas propios especificados por $\varphi$ (punto iii. anterior).

4. Por último, es necesario dar una interpretación empírica de la teoría axiomatizada -que no es un requisito estrictamente formal, pero que mostrará la utilidad del método teórico-conjuntista de Suppes-.

Con este método, según Suppes, estamos en condiciones de reconstruir una teoría empírica y de investigar las consecuencias deductivas de tal definición. Suppes cree que su axiomatización permitirá hacer las preguntas adecuadas, semejantes a las que se hacen en la matemática moderna (como acerca de qué tipo de teoremas pueden ser probados), y abordar cuestiones importantes como el problema del reduccionismo teórico. Los modelos de las teorías empíricas son estructuras conjuntistas que satisfacen las leyes de una teoría, que son las condiciones restrictivas especificadas por (según el punto iii.), y que son interpretadas empíricamente (según el paso 4.). Para Suppes, esto funcionará muy bien con la física:

Es bastante simple ver cómo un modelo físico real, en el sentido de los físicos de la mecánica clásica de partículas ${ }^{5}$, está relacionado con este sentido teórico-conjuntista de modelos. Simplemente podemos considerar que el conjunto de partículas es, en el caso del sistema solar, el conjunto de cuerpos planetarios. Otra posibilidad ligeramente más abstracta es tomar el conjunto de partículas como el conjunto de centros de masa de los cuerpos planetarios. Esto generalmente ejemplifica la situación. El conjunto abstracto teórico de una teoría tendrá entre sus partes un conjunto básico que consistirá en los objetos que, normalmente, se consideran como constituyentes del modelo físico [...] Parece que no sirve de nada discutir sobre qué uso de la palabra 'modelo' es el primario o el más apropiado en las ciencias empíricas. Mi propia opinión en este artículo es que el uso conjuntista es el más fundamental (Suppes 1960, p. 291).

La tarea principal de la axiomatización de una teoría será entonces la definición de un predicado conjuntista. Tal predicado define una clase de modelos para la teoría. Las estructuras especificadas en $x$, de ' $x$ es un sistema syss $_{d e f} \varphi(x)^{\prime}$, son las que satisfacen las condiciones definicionales o 'tipificaciones' (punto ii.) y así son las candidatas a ser modelos de la teoría (Suppes 1960, p. 353). Son entidades de las cuales cabe preguntarse si cumplen las leyes específicas de la teoría. Si satisfacen esas tipificaciones se llamarán 'realizaciones posibles' de la teoría. Si dichas estructuras además satisfacen los axiomas o condiciones restrictivas (las leyes en sentido propio, punto iii.), serán sus 'realizaciones efectivas' o modelos, en sentido propio.

Las teorías son entonces:

La axiomatización de la mecánica clásica de partículas se encuentra en Suppes (1957, pp. 291-305). 
[...] sistemas [o estructuras] ideales constituidos, mediante definición, por conjuntos de enunciados o leyes teóricas que afirman o niegan que un sistema real empírico [...] está o no de acuerdo con el [...] modelo teórico o sistema definido (Cuevas 2016, 42).

Esta perspectiva pone énfasis en la noción de modelo - dada por Tarski-, contrariamente a la perspectiva clásica que caracterizaba a las teorías como conjuntos de enunciados y que ponía el énfasis en los axiomas y reglas de correspondencia (formalización) para su reconstrucción: teorías como sistemas axiomáticos.

Para el enfoque semanticista, una teoría científica se caracterizará entonces por estar determinada por una clase de modelos, su método axiomático 'semi-formal' (teórico-conjuntista) se entenderá como suficiente para identificar la familia de modelos que le corresponde a dicha teoría. Las formulaciones lingüísticas de la teoría quedan asumidas y supeditadas a esa identificación con la clase de modelos (Torretti 1990, 109130). Aunque los modelos de la teoría se determinen a través de un conjunto de axiomas, la identidad de la teoría -para Suppes- no depende de la formulación lingüística en cuestión, sea una u otra. La clase de modelos podrá ser descrita, por ejemplo, mediante enunciados diferentes en lenguajes distintos. Incluso, conjuntos de enunciados diferentes pueden serlo de una misma teoría. Por ello, el tipo de formalización al que se recurre es el de la teoría básica de conjuntos que, según Suppes, resulta un recurso formal más rico y no anclado exclusivamente a una reconstrucción a partir de la lógica de primer orden, como lo hacía la 'Concepción Heredada'.

\section{Observaciones a la interpretación de Suppes}

Esta interpretación del método axiomático por parte de Suppes supera algunos escollos de la aplicación del enfoque clásico. Sin embargo, se pueden hacer las siguientes tres observaciones, formuladas como crítica, que pueden resultar importantes respecto de la interpretación de las estructuras de las teorías y de la noción de modelo que tiene la concepción semanticista.

\subsection{La semántica estática}

La primera es respecto de la semántica en cuestión. La propuesta de Suppes no deja de ser una perspectiva semántica modelo-teorética-tarskiana -nos permitimos aquí hacer esta redundancia-. Esto quiere decir que, aunque se construya una formalización con teoría básica de conjuntos (como herramienta de cálculo), su semántica es una semántica de modelos y por esto asume una teoría de la verdad específica-como se señaló en la subsección 2.2.1-. Sin embargo, actualmente existen otros enfoques semánticos muy desarrollados y más ricos en lógica-matemática como, por lo pronto, los provenientes del enfoque de teoría de juegos.

El punto de vista semántico de la teoría de modelos ha sido cuestionado -principalmente desde la década de los 90- por permanecer anclado a una visión 'estática' de los conceptos lógicos-matemáticos fundamentales, como los de 'verdad', 'teoría' y 
'modelo'; criticados especialmente por Johan van Benthem (1994; 2014). El enfoque ‘dinámico' de los sistemas lógicos (teoría de juegos) se desarrolla a partir de una crítica al concepto de verdad lógica tarskiano. Para van Benthem, muchas nociones cognitivas y lógicas centrales tienen un carácter dual: existe una interacción entre contenidos estáticos y acciones dinámicas. Por ejemplo, 'juicio' significa tanto una acción intelectual como el contenido de esa acción. En efecto, 'razonamiento' (o 'conocimiento', etc.) denota tanto un proceso intelectual como también se refiere a sus productos. Otro ejemplo es el uso corriente del término 'lenguaje natural', que puede entenderse como una estructura matemática estática de palabras y reglas o bien como una actividad dinámica social (van Benthem 1994, pp. 108-109). Este enfoque semántico-pragmatista intenta dar cuenta de que las significaciones de los conceptos lógicos-matemáticos fundamentales son construcciones abstractas que se dan en una actividad lingüística concreta, dentro del marco de la práctica de la demostración o prueba lógico-matemática. Por ejemplo, contrariamente a la idea de Tarski, este enfoque sostendrá que la verdad es más bien una construcción teórica que depende de cómo han sido relacionados los símbolos en la estructura en la que participan, o cómo se establecen las relaciones entre estructuras o sistemas de inferencia. Así, toda definición lógica tiene una dimensión procedural u operacional, determinada por la acción de un agente, de la cual la semántica en cuestión tiene que dar cuenta (Keiff 2009; Lorenz 2010). De esta manera, los sistemas formales que se construyen son diferentes, ya que tienen que explicar ese dinamismo inferencial. En cambio, el axiomatismo de la perspectiva semanticista mantiene -sin dudas- una teoría de la verdad por correspondencia para la relación teoría-modelomundo, asumiendo todas las consecuencias realistas que se siguen de ella.

Si bien es cierto que no se han desarrollado hasta ahora aplicaciones formales dinámicas - de manera sistemática- para el análisis de teorías empíricas, las críticas actuales realizadas en los ámbitos de la lógica y de la filosofía del lenguaje han mostrado tanto las limitaciones lógicas como epistemológicas de la semántica de modelos y de los recursos axiomáticos basados en enfoques 'estáticos', especialmente de la lógica y de la matemática ( $c f$. Cellucci 1993a; Martin-Löf 1996; Rahman y Clerbout 2013; Rahman et al., 2018; Redmond y Lopez-Orellana 2018). Para la filosofía de la ciencia, estas críticas provenientes de la lógica y la filosofía del lenguaje pueden resultar muy sugerentes, por ejemplo, para la revisión de las perspectivas del realismo científico o de la representación científica.

\subsection{Las condiciones restrictivas y el lenguaje para la axiomatización}

La segunda observación es respecto de las condiciones restrictivas -o leyes de la teoríaque se sostienen para el método axiomático de este enfoque (señaladas en el anterior punto iii, subsección 2.2.2). Díez y Moulines (2016) señalan que, si bien el lema de la concepción semántica que funda Suppes es 'presentar una teoría es presentar una clase de modelos, no de axiomas', es importante saber que con ello no se pretende prescindir de los enunciados o formulaciones lingüísticas. Los recursos lingüísticos no son superfluos: "para determinar o definir una clase de modelos hace falta un lenguaje” (Díez y Moulines 2016, p. 351). Esto es, para la caracterización metateórica 
la formulación lingüística axiomática es solo el medio necesario para la determinación de los modelos. Según los semanticistas, es posible usar cualquier tipo de lenguaje, que sea la lógica de primer orden o no es una cuestión trivial. Sin embargo, a pesar de esta advertencia, debe reconocerse que las condiciones restrictivas solo pueden expresarse en un lenguaje que viene determinado de alguna manera por la estructura o sistema en cuestión, especificada en ' $x$ es un sistema syss $_{\text {def }} \varphi(x)^{\prime}$. En este sentido, si bien la identidad de la teoría puede no depender de la formulación lingüística, el tipo de formulación o axiomatización que se necesita generalmente está determinado con anterioridad por una base lógica que no es trivial. Para especificar lo que se quiere decir aquí, recordemos la estructura básica de una axiomatización (columna izquierda del siguiente cuadro):

\begin{tabular}{|l|l|}
\hline $\begin{array}{l}\text { Sistema axiomático formal } \\
\text { (cerrado) }\end{array}$ & $\begin{array}{l}\text { Sistema axiomático semi-formal de una teoría factual } T \\
\text { (abierto) }\end{array}$ \\
\hline 0. Lógica subyacente & 0. Lógica subyacente \\
\hline 1. Vocabulario: & 1. Vocabulario: \\
1.1 Símbolos lógicos & $\begin{array}{l}\text { 1.1 Símbolos lógicos } \\
\text { 1.2 Términos primitivos }\end{array}$ \\
1.2 Términos pre-teóricos \\
1.3 Términos definicos de las leyes teóricas \\
1.4 Términos observacionales (de las generalizaciones o \\
leyes empíricas)
\end{tabular}

\section{Reglas de Correspondencia \\ (reglas como 'principios de interpretación empírica'; con un estatus diferente fuera del cálculo del sistema).}

Cuadro 1. Representación general de la estructura básica de cualquier axiomatización. Sistema axiomático cerrado, columna izquierda. Sistema axiomático abierto, de una teoría empírica o factual, columna derecha.

A la izquierda se representa la estructura de un sistema axiomático formal (lógicomatemático), entendido como un conjunto de proposiciones cerrado bajo el operador de consecuencia. A la derecha se ha incluido, solo como comparación, la estructura de un sistema axiomático semi-formal (Hempel 1970), en la interpretación de la concepción clásica más básica de las teorías, como un conjunto de proposiciones abierto en relación con la inclusión y aplicación de las reglas de correspondencia (ubicadas fuera del cuadro para la comparación); la concepción semanticista moldea esta base para la inclusión del aparato conjuntista, y que su versión estructuralista refina para dar cuenta de las llamadas 'redes teóricas' e incluir la 'aserción empírica' (Torretti 1990, p. 116; Lorenzano 2008). 
Lo que no logró ver Suppes, y que los semanticistas posteriores olvidaron, es que la estructura lingüística de una axiomatización no solo está compuesta por los elementos de 1., 2., 3., 4. y 5. del Cuadro 2.1. Como demuestran Gregorio Klimovsky y Guillermo Boido (2005, pp. 135-148), todo sistema axiomático, incluso los 'semi-formales' de las teorías factuales, se compone también de una 'lógica subyacente' (0.) que no se explicita. Este tipo de lógica comprende los conceptos y principios lógicos -por lo pronto, principio de identidad, (principio de no-contradicción), (principio del tercero excluido), ex falso quodlibet (o principio de explosión) y principio de monotonicida-que son básicos y en los cuales se apoya y define todo el resto de formulación lingüística, esto es, aquello que posteriormente llamamos 'lenguaje lógico', en sentido propio. En efecto, prácticamente toda la reconstrucción de teorías por parte de la concepción clásica del positivismo lógico es en lógica de primer o de segundo orden (extensión de la primera) porque su 'lógica' subyacente se compone de principios y nociones de primer orden. Es más, la definición de la verdad y la noción de modelo (nociones semánticas) ofrecidas por Tarski lo son para un lenguaje de lógica de primer orden, con el que intenta mejorar las pruebas de consistencia y completud semántica de esta lógica. La noción de modelo que Tarski propuso aplicar a las teorías empíricas tiene esta base y es la que asume Suppes en su concepción semanticista, con sus consecuentes modificaciones. Si bien es cierto que en toda la perspectiva semanticista no se hace hincapié en los recursos lingüísticos -en los axiomas o leyes fundamentales ('principios guías' según Moulines 1978)- para su análisis o reconstrucción metateórica de las teorías empíricas, para definir sus modelos sí necesitan de un lenguaje. Generalmente su forma de caracterizar las teorías empíricas es definiendo predicados de segundo orden en términos conjuntistas, o sea, aplicando la teoría intuitiva de conjuntos donde las cuantificaciones existenciales involucradas son de lógica de segundo orden, esto es, inevitablemente dentro de un paradigma lógico clásico.

Por ejemplo, para el caso de las reconstrucciones teóricas de los metaestructuralistas seguidores de Suppes, cabría preguntarse qué tipo de lenguaje es el más conveniente para una reconstrucción, o si corresponde solo un tipo de formulación lingüística para una determinada teoría empírica. Obviamente, estas preguntas son triviales para ellos. Pero lo que debemos comprender es que la teoría de conjuntos, que permite a esta concepción realizar formalizaciones efectivas, es aplicada aquí como una herramienta auxiliar para un determinado cálculo (sintaxis) donde previamente se ha seleccionado ya de partida una semántica específica (Cassini 2013). Una revisión de lo que significa un sistema axiomático se vuelve necesaria entonces ( $c f$. Fernandes 2017, Lopez-Orellana 2019). La pregunta vuelve a ser: ¿qué sintaxis y qué semántica hemos escogido para reconstruir o analizar una teoría de la realidad empírica? También podemos preguntar ¿esa parcela del mundo de la cual habla la teoría se 'ajusta' de alguna manera a mi reconstrucción formal? ¿los modelos definidos por ese lenguaje determinado (axiomas) logran realizar efectivamente la teoría acerca de dicha parcela del mundo? No es difícil darse cuenta de por qué casi todas las reconstrucciones y análisis de teorías empíricas realizadas en este enfoque provengan de teorías de la física o la química, que son altamente matematizables, y muy pocas incursiones se han realizado para la biología u otras ciencias en las que (toda) la explicación científica carece de leyes o axiomas en sentido propio (Lorenzano 2008). Como dicen Morrison y Morgan (1999, p. 2): 
Tal vez no sea sorprendente que gran parte de la literatura temprana sobre la estructura de la teoría y los modelos en la filosofía de la ciencia tome a la física como punto de partida. Las teorías físicas no solo son altamente matemáticas, sino que ciertamente son más fáciles de plasmar en una forma axiomática en comparación a las teorías de otras ciencias.

Es difícil exigir a las teorías de la biología -por ejemplo- leyes como condiciones restrictivas no lógicas de las estructuras que permitan determinar una representación modelística efectiva.

Además, resulta interesante revisar cómo y por qué da Costa y French (2003), dentro del propio enfoque semanticista, intentan otro camino recurriendo a la lógica paraconsistente, en cuya lógica 0 no se incluye el principio clásico ex falso quodlibet. $\mathrm{Su}$ propuesta es lograr un mejor abordaje del carácter local de la producción del conocimiento y la naturaleza fragmentada de las representaciones empleadas para el razonamiento científico. Para ellos, todas las representaciones fenoménicas son crucialmente incompletas y abiertas, por lo que deben considerarse no como verdaderas -en el sentido de 'correspondencia'-sino como parcialmente verdaderas o aproximadamente verdaderas -o al menos, conteniendo algún elemento de verdad- (da Costa y French 2003 , p. 4). Según esto, algunos conceptos y principios lógicos fundamentales, como la verdad y la no-contradicción, deben cambiarse o eliminarse.

\subsection{Acerca de los conceptos de 'sistema axiomático', 'sistema formal' y 'método axiomático'}

Como se señaló anteriormente, otra cuestión interesante que debe revisarse del análisis y caracterización teórica de Suppes, y de los semanticistas posteriores, es qué entiende por sistema axiomático, sistema formal y método axiomático.

La revisión más pertinente a este respecto se encuentra en Carlo Cellucci (1993a). Cellucci afirma que los filósofos de la ciencia han olvidado que otra de las consecuencias del teorema de incompletitud de Kurt Gödel es que, aparte de llevar al fracaso una gran parte del programa de David Hilbert, el concepto de sistema formal como sistema cerrado es inadecuado para las matemáticas y la lógica; aunque "se continúa usando como si no hubiera un teorema de incompletitud" (Cellucci 1993, p. 206). Recuérdese que bajo el concepto de 'sistema axiomático' y de 'método axiomático' está la idea de que las teorías matemáticas son deducciones de los 'primeros principios' (lógicos) (Lopez-Orellana 2019, pp. 285-288). Dada esa inadecuación del concepto de sistema formal, Cellucci aboga por la necesidad de reformulación del concepto de lógica: que deberá ser entendida ahora como teoría de la comunicación de los procesos de inferencia ${ }^{6}$

6 Cellucci (1993a) llama a este nuevo paradigma de lógica 'lógica computacional', porque los procesos y la comunicación son una de las principales preocupaciones de la informática. Cellucci aplica su perspectiva al lenguaje de programación Prolog, que lo piensa como un lenguaje lógico de derecho propio y con un alcance más amplio que la perspectiva lógica 
(Cellucci 1993a, pp. 206-207). Esta definición puede identificarse dentro del enfoque dinámico de la lógica ${ }^{7}$, que se ha mencionado en la subsección 2.3.1.

Para justificar su perspectiva, Cellucci se remonta a las dos formas diferentes del método científico que se desarrollaron en la filosofía antigua, especialmente para las matemáticas: 1) el método axiomático, en el que se supone que las teorías científicas son sistemas cerrados; $y, 2)$ el método analítico, pero en el sentido platónico de 'reducción' -que veremos a continuación-, en el que las teorías se consideran sistemas abiertos. De acuerdo con la terminología de van Benthem (1994), el concepto de teoría que se sostiene en 1) es estático, esto es, concibe una teoría científica como un producto 'acabado'. Por ello, el método que le corresponde es el método axiomático (Cellucci 2017 , p. 173) esto significa que todos los problemas de una ciencia se resuelven encontrando deducciones de sus principios. Según Cellucci (1993a, pp. 214-215), se suele identificar dicho método con Aristóteles (cf. An. Post. I 1, 71a 1-10; 1960) que, si bien lo presentó solo como un método para la enseñanza de la ciencia-más que en un método para hacer ciencia-, cometió el error de pensar que la mayoría de las ciencias, incluyendo las matemáticas, eran sistemas cerrados que representan una realidad cerrada y totalmente determinada. A pesar de que el aspecto didáctico en el cual Aristóteles concibe este método es fácil de descifrar, los lógicos modernos lo identificaron como el método matemático por antonomasia (Cellucci1993b).

En cambio, según Cellucci, el método analítico -no en el sentido geométrico posterior a Platón- pone en el centro de la lógica a los procesos de inferencia y comunicación. Cellucci retorna a Platón y afirma que la dialéctica-como 'dia-lógica'juega un papel importante, la entiende como un método auxiliar al método analítico por el cual las hipótesis son elegidas. La dialéctica no es más que el proceso en el cual dos agentes dialogan para llegar a un estado de acuerdo, desde un punto de partida que es un estado de desacuerdo o incertidumbre:

La condición inicial representa un estado de incertidumbre sobre el problema mientras que la condición final representa un estado de certeza. El paso del estado de incertidumbre al estado de certeza corresponde a un avance del conocimiento (Cellucci 1993a, p. 208).

En este sentido, la dialéctica (o dialógica) podrían incluirse como parte del método analítico. Es por esto que:

matemática clásica. En Prolog, los predicados analizados para la manipulación de la base de datos -v. g., 'afirmar' y 'retractarse'- hacen que los axiomas y las reglas de inferencia puedan cambiar en el curso de la prueba. Es decir, los principios de una prueba axiomática son modificados de tal manera que las relaciones que se mantienen en un momento determinado pueden dejar de mantenerse en otro momento.

Para un argumento más acabado al respecto, véase López y Redmond (2018, pp. 229-231). 
Platón (en Menón 86d-87d, Fedón 99d-101e, República VI 509d511e, VII 525b-527b, 531c-533e) [identificó] el método matemático con un procedimiento de resolución de problemas que, a diferencia del método axiomático, no parte de principios dados sino que reduce el problema a una hipótesis de tal manera que, si la hipótesis se satisface, entonces el problema puede ser resuelto: por lo tanto el procedimiento es una búsqueda hacia atrás (Cellucci 1993a, p. 207).

De esta manera, el análisis es un proceso potencialmente infinito de búsqueda de mejores hipótesis, por el cual se entiende que toda ciencia está siempre 'en construcción'. El método analítico no es más que la reducción de un problema a otro, en el cual las hipótesis se entienden no como proposiciones básicas (como fundamentos de todas las matemáticas), sino solo como condiciones de solución de un problema específico. $\mathrm{Y}$ en él no cabe distinguir entre teorías como sistemas abiertos o cerrados. Todas las teorías son sistemas abiertos y dinámicos.

Cellucci (1993a, p. 211) culpa principalmente a Gottlob Frege y a David Hilbert de caracterizar la lógica-matemática solo como un estudio del método axiomático en el cual cabe únicamente la consideración de los sistemas cerrados ${ }^{8}$. A Cellucci le resulta curioso ver que Hilbert y la mayoría de los matemáticos -incluso en la actualidad, salvo los intuicionistas-persisten en que los sistemas formales de la lógica-matemática, y los sistemas de objetos que representan, solo pueden ser sistemas cerrados. Para Cellucci, comprender la matemática a través del método analítico -es decir, como un procedimiento (perfectible) de ensayo y error para la búsqueda de hipótesis que puedan fundamentarse (potencialmente) cada vez mejor-nos indica que la única forma de concebir el conocimiento científico -incluso el formal- es simplemente como noabsoluto y como una búsqueda continua de hipótesis. En efecto:

[...] un enfoque axiomático sólo sería posible si el conocimiento fuera un sistema cerrado, es decir, un patrimonio estable y garantizado de verdades basado en principios dados de una vez por todas (Cellucci 1993a, p. 209).

Entonces, para Cellucci, los lógicos, matemáticos y filósofos de la ciencia deberían advertir que el teorema de incompletitud de Gödel muestra de forma directa que cualquier sistema formal, seleccionado para cualquier rama de las matemáticas, es intrínsecamente provisional y debe admitir siempre extensiones apropiadas. Por lo

$8 \quad$ Según la historia de la lógica, Hilbert “estaba convencido de que el método axiomático pertenecía a la lógica. Para él, la lógica de su época era una ciencia que ya había avanzado lo suficiente y se demostraba en el mismo hecho de habernos dotado de un método [la axiomática] capaz de revelar, por ejemplo, que el problema del infinito no se sustentaba en ningún significado intuitivo y que una investigación más detallada al respecto tampoco tenía sentido: "por todas partes solo hay cosas finitas" (Hilbert 2005c, p. 1160) [...] [incluso] respecto de las ciencias naturales [...] debemos asumir con estas teorías que únicamente hay cosas finitas: no hay velocidad infinita ni ninguna fuerza o efecto que se propague infinitamente rápido por el espacio" (Lopez-Orellana 2019, p. 299). 
tanto, el concepto de sistema formal como sistema axiomático (cerrado) es incapaz de representar el proceso matemático. Cellucci muestra detalladamente esta crítica en el capítulo 12 de su libro Rethinking Knowledge. The Heuristic View (2017, pp. 149-173).

Si esto ocurre con las matemáticas y la lógica, lo que se le puede plantear a Suppes y al semanticismo vuelve a ser entonces ¿cómo se ajusta una reconstrucción (y análisis) formal (axiomático) intrínsecamente estático y cerrado a sistemas intrínsecamente abiertos, como son las teorías de las ciencias empíricas? Es más, los sistemas de fenómenos de los que las teorías intentan dar cuenta son también intrínsecamente abiertos. Los autores de la Concepción Heredada y los semanticistas llaman a sus reconstrucciones axiomáticas de teorías científicas 'sistemas axiomáticos semi-formales o abiertos', tal como ha sido representado en el Cuadro 2.1 de la subsección anterior (2.3.2). Lo que hacen es una reconstrucción semi-formal a partir de un recurso matemático, que es el método axiomático más el aparato conjuntista, forzando la estructura de un sistema axiomático para introducir términos y proposiciones empíricas de las cuales pueden probarse los postulados e hipótesis de esas teorías. En palabras de Cellucci, confunden el concepto de sistema axiomático con el de sistema abierto y con ello suponen para el método axiomático una aplicabilidad mayor de la que realmente tiene. En efecto, vuelve a ser pertinente entonces preguntarse ¿por qué casi todos sus análisis y reconstrucciones lo son de teorías de la física o la química y muy pocos son sus intentos para ciencias -como la biología- en las que se carece de leyes o axiomas?

Además de esto, siguiendo a Cellucci (2017, Cap. 13), con un aparato axiomático como este -a pesar de su definición de 'semi-formal'- los semanticistas solo pueden poner énfasis en las estructuras teóricas que dan cuenta -de alguna manera- de cierta estructura de la realidad, esto es, solo ponen énfasis en el aspecto estático de los sistemas fenoménicos (como el revelado de una foto). Como sabemos, los semanticistas sostienen -aunque no lo hagan explícito- que un modelo puede ser usado con precisión para representar un fenómeno solo si tiene una subestructura isomórfica a ese fenómeno (van Fraassen 2008, p. 309). Pero,

[...] los sistemas físicos tienen propiedades tanto estructurales como de comportamiento. El comportamiento se refiere a las transiciones de estado y a las propiedades dinámicas -operaciones y sus relaciones-. Los modelos deben ser capaces de expresar cómo y cuándo ocurren los cambios en las entidades y sus relaciones. [P] ero las estructuras son incapaces de expresarlo (Cellucci 2017, p. 183).

Para Cellucci, los semanticistas serán entonces incapaces de dar cuenta de los modelos científicos porque en su noción básica de modelo solo ponen énfasis en su aspecto estático, en su estructura. Para ellos, un modelo es una estructura abstracta, por lo tanto, un objeto matemático o -al menos- una estructura que puede reconstruirse en una representación matemática. Sin embargo, para Cellucci, tanto las teorías como los modelos deben ser entendidos como sistemas abiertos y dinámicos; esto es, como sistemas de hipótesis no acabados que pueden apelar a otros sistemas para cubrir sus lagunas (Cellucci 2017, p. 179). Obviamente, tal definición de 'modelo' (también de 'teoría') no podrá provenir de una perspectiva semántico-axiomática. 


\section{Consideraciones finales}

El enfoque semanticista fundado por Suppes es, sin duda, una de las perspectivas más influyentes y desarrolladas en filosofía de la ciencia. La revisión que hemos realizado responde a la necesidad de despejar algunos de sus puntos centrales. Esta concepción ha moldeado la noción filosófica básica y representacional de 'modelo' científico. En efecto, la concepción filosófica dominante de los modelos científicos los ha entendido a partir del concepto de representación científica (Cassini 2016): los modelos son representaciones idealizadas o simplificadas de los fenómenos. Por supuesto, el concepto de representación tiene una larga tradición en filosofía, refiere a la forma en la que accedemos y conocemos el mundo. Sin embargo, es con Suppes que surge la idea contemporánea del realismo científico de que la representación es la función más importante que tiene un modelo ( $c f$. van Fraassen 1980, 1987; Kitcher 1993; Giere 1988, 1999; Morrison 1999; Suárez 2019). Dicha función establece la relación de correspondencia entre la estructura de las teorías y la estructura de los fenómenos del mundo. De esta manera, es con Suppes que se establece una estrecha relación entre las nociones de modelo y representación.

La crítica que hemos moldeado, desde un punto de vista formal, ofrece argumentos a favor de la inadecuación de la noción representacional de modelo aplicada a las teorías y modelos científicos. Actualmente, las propuestas que se desarrollan en filosofía de la ciencia han tomado distancia de cualquier consideración de la noción de modelo como estrictamente semántica y basada exclusivamente en el concepto de representación; poniéndose énfasis en nociones pragmáticas como 'uso de modelos' o 'teorías como herramientas para la construcción de modelos' (v .g., (Godfrey-Smith 2006; Suárez 2004; Suárez y Cartwright 2008; Knuuttila 2011, 2014, 2018; Mäki 2009). Esta crítica ofrece otros elementos a favor de una superación de la noción de modelo estrictamente semántica o axiomática.

Una propuesta de reconstrucción formal de las teorías de las ciencias empíricas en términos del método analítico propuesto por Cellucci, además de ser interesante, podría dar un ejemplo o una respuesta más acabada a la pregunta acerca de cómo serían posibles reconstrucciones de teorías de ciencias en las que se carece de leyes o axiomas -como la biología o la economía-.

\section{Referencias bibliográficas}

Aristotle (1960), Posterior Analytics. Topica, Vol. 391 of Loeb Classical Library. Harvard: Harvard University Press.

Autor (2018)

Balzer, W., Moulines, C. U. \& Sneed, J. D. (1987), An Architectonic for Science. The Structuralist Program, Vol. 186 of Synthese Library. Dordrecht: Springer Netherlands.

Carnap, R. (1956), The methodological character of theoretical concepts, Vol. 1. Minnesota: University of Minnesota Press. 
Cassini, A. (2013), El juego de los principios. Una introducción al método axiomático. Buenos Aires: A-Z Editora.

Cellucci, C. (1993a), From closed to open systems, in J. Czermak, ed., Proceedings of the 15th International Wittgenstein Symposium. Wien: Hölder-PichlerTempky; pp. 206-220.

(1993b), Gli scopi della logica matematica, in Peano e i fondamenti della matematica. Atti del Convegno Modena 22-24 ottobre 1991. Mucchi: Modena; pp. 73-138.

(2017), Rethinking Knowledge. The Heuristic View, Vol. 4 of European Studies in Philosophy of Science. Cham: Springer.

Cuevas, A. (2016), Organización y estructura del conocimiento cientifico. Buenos Aires: Eudeba.

da Costa, N. C. A. \& S. French (2003), Science and Partial Truth. A Unitary Approach to Models and Scientific Reasoning. New York: Oxford University Press.

Dalla Chiara, M. L. \& G. Toraldo di Francia (1973), A logical analysis of physical theories, Rivista di Nuovo Cimento 2 (3): 1-20.

Díez, J. A. \& C. U. Moulines (2016), Fundamentos de filosofia de la ciencia. Barcelona: Editorial Ariel.

Fernandes, D. P. (2017), Logical Systems: On the Concept, Expressive Power and Expressiveness Characterizations, $\mathrm{PhD}$ thesis, Instituto de Estudios Sociales de la Ciencia y la Tecnología. Salamanca: Universidad de Salamanca.

Frigg, R. (2006), Scientific representation and the semantic view of theories, Theoria 21 (1): 49-65.

Giere, R. (1988), Explaining Science: A Cognitive Approach. Chicago: University of Chicago Press.

(1999), Science without Laws. Chicago: University of Chicago Press.

Hempel, C. G. (1962), Deductive-Nomological vs. Statistical Explanation. Minneapolis: University of Minnesota Press.

(1970), On the 'standard conception' of scientific theories, Vol. IV Analyses of Theories and Methods of Physics and Psychology. Minneapolis: University of Minnesotta Press.

Hodges, W. (2014), Tarski's truth definitions, Standford Encyclopedia of Philosophy. URL: https://plato.stanford.edu/entries/tarski-truth/

Keiff, L. (2009), Dialogical logic, Standford Ecyclopedia of Philosophy. URL: http:// plato.stanford.edu/entries/logic-dialogical/

Klimovsky, G. \& G. Boido (2005), Las desventuras del conocimiento matemático. Buenos Aires: A-Z Editora.

Lopez-Orellana, R. (2019), El enfoque epistemológico de David Hilbert: el a priori del conocimiento y el papel de la lógica en la fundamentación de la ciencia, Principia. An Internacional Journal of Epistemology 23 (2): 279-308. 
Lorenz, K. (2010), Logic, Language and Method. On Polarities in Human Experience. New York: De Gruyter.

Lorenzano, P. (2008), Lo a priori constitutivo en la ciencia y las leyes (y teorías) científicas, Revista de Filosofía 33 (2): 21-48.

(2013), The semantic conception and the structuralist view of theories: A critique of suppe's criticisms, Studies in History and Philosophy of Science Part A 44 (4): 600-607.

Martin-Löf, P. (1996), On the Meanings of the Logical Constants and the Justifications of the Logical Laws, Nordic Journal of Philosophical Logic 1 (1): 11-60.

Morrison, M. \& M. S. Morgan (1999), Introduction, en M. Morrison y M. S. Morgan, Models as mediators. Perspectives on natural and social sciences, Cambridge: Cambridge University Press; pp. 1-9.

Morselli, M. (1984), Amedeo Avogadro: A Scientific Biography. Dordrecht: D. Reidel Publishing Company.

Moulines, C. U. (1978), Forma y función de los principios-guía en las teorías físicas, Crítica 10: 59-88.

(2011), El desarrollo moderno de la filosofía de la ciencia. México: UNAM, Instituto de Investigaciones Filosóficas.

Rahman, S. \& N. Clerbout (2013), Constructive type theory and the dialogical approach to meaning, The Baltic International Yearbook of Cognition, Logic and Communication 8: 1-72.

Rahman, S., Z. Mc Conaughey, A. Klev \& N. Clerbout (2018), Immanent Reasoning or Equality in Action. A Plaidoyer for the Play Level, Vol. 18 of Logic, Argumentation and Reasoning. Dordrecht: Springer International Publishing.

Redmond, J. \& Lopez-Orellana, R. (2018), Lógica clásica y esquizofrenia: por una semántica lúdica, Revista de Filosofia 74, 214-241.

Sneed, J. D. (1971), The Logical Structure of Mathematical Physics. Dordrecht: D. Reidel.

Stegmüller, W. (1970), Theorie und Erfahrung, Vol. 2 of Probleme und Resultate der Wissenschaftstheorie und Analytischen Philosophie. Berlin: Springer-Verlag.

(1973), Theorienstrukturen und Theorien-Dynamik. Zweiter Halbband Theorienstrukturen und Theoriendynamik, Vol. 2/2 of Theorie und Erfahrung. Berlin: Springer-Verlag.

Suppe, F. (1989), The Semantic Conception of Theories and Scientific Realism. Chicago: University of Illinois Press.

Suppes, P. (1954), Some remarks on problems and methods in the philosophy of science, Philosophy of Science 21 (3): 242-248.

(1957), Introduction to Logic. London: Van Nostrand Reinhold Company.

(1960), A comparison of the meaning and uses of models in mathematics and the empirical sciences, Synthese 12 (2/3): 287-301. 
(1962), Models of data, in E. Nagel, P. Suppes \& A. Tarski, eds., Logic, Methodology and Philosophy of Science: Proceedings of the 1960 International Congress. Stanford: Stanford University Press; pp. 252-261.

(1970), Set-Theoretical Structures in Science. Stanford: Stanford University Press.

(1974), The axiomatic method in the empirical sciences, in L. Henkin, ed., Proceedings of the Tarski Symposium, Vol. XXV. Providence: American Mathematical Society; pp. 465-479.

(1989), Representation theory and the analysis of structure, Philosophia Naturalis 25: 254-268.

(1993), Models and Methods in the Philosophy of Science. Selected Essays. Dordrecht: Kluwer.

Tarski, A. (1935), Der Wahrheitsbegriff in den formalisierten Sprachen, Studia Philosophica (1): 261-405.

(1936), Über den Begriff der logischen Folgerung, in Actes du Congrès International de Philosophie Scientifique. Sorbonne, Paris, 1935, Vol. VII: Logique of Actualités Scientifiques et Industrielles, 394, Paris: Hermann \& Cie, Édi- teurs; pp. 1-11.

(1944), The semantic conception of truth and the foundations of semantics, Philosophy and Phenomenological Research (4): 341-376.

(1977), Introducción a la lógica y a la metodología de las ciencias deductivas. Madrid: Espasa-Calpe.

(1983), The concept of truth in formalized languages. Indianapolis: Hackett Publishing; pp. 152-278.

Torretti, R. (1990), Creative Understanding. Philosophical Reflections on Physics. Chicago and London: The University of Chicago Press.

van Benthem, J. (1994), General dynamic logic, Studies in Logic and Computation Series. Oxford: Clarendon Press; pp. 107-140. (2014), Logic in Games. Massachusetts: MIT Press.

van Fraassen, B. C. (1980), The Scientific Image. Oxford: Clarendon Press. (2008), Scientific Representation: Paradoxes of Perspective. Oxford: Clarendon Press.

Worrall, J. (1989), Structural realism: The best of both worlds?, Dialectica 43: 99124. 